\title{
HBME-1 and antithrombomodulin in the differential diagnosis of malignant mesothelioma of pleura
}

\author{
A D Kennedy, G King, K M Kerr
}

\begin{abstract}
Aims-To determine the usefulness of antibodies HBME-1 and antithrombomodulin in the differential diagnosis of malignant mesothelioma of the pleura.

Methods-Using microwave antigen retrieval and streptavidin-biotin complex horseradish peroxidase immunohistochemistry the above antibodies were used to stain sections of 57 malignant mesotheliomas, 17 reactive pleural hyperplasias, 23 cases of carcinoma metastatic in pleura, 20 primary ovarian cell carcinomas, and 20 primary renal cell carcinomas.

Results-Eighty six per cent of mesotheliomas and $82 \%$ of reactive mesothelial hyperplasias stained strongly with HBME-1. However, $48 \%$ of carcinomas metastatic to pleura also stained, as did all serous ovarian carcinomas. Seventy two per cent of mesotheliomas and $24 \%$ of reactive mesothelial hyperplasias stained strongly with the antithrombomodulin antibody; $86 \%$ and $88 \%$, respectively, of these cases showed staining of any type. While $26 \%$ of metastatic carcinomas showed some staining with antithrombomodulin, only one third of these $(9 \%)$ showed strong, yet focal, staining. Of 40 ovarian and renal carcinomas only two (5\%) showed any staining with antithrombomodulin.

Conclusions-HBME-1, although a sensitive mesothelial marker, is not sufficiently specific to be useful diagnostically, as almost half of carcinomas metastatic to pleura also stained positive. Antithrombomodulin is also a sensitive mesothelial marker and is sufficiently specific to be a useful discriminator, positively identifying, in appropriate circumstances, the mesothelial nature of a cell population. (F Clin Pathol 1997;50:859-862)
\end{abstract}

Keywords: HBME-1; thrombomodulin; mesothelioma; differential diagnosis

Pathology, Aberdeen

Royal Infirmary and

University Medical

School, Foresterhill,

Aberdeen AB25 2ZD, UK

Correspondence to: Dr Kennedy.

Accepted for publication 15 July 1997 A particular issue has been the lack of a specific marker for mesothelial cells in formalin fixed, paraffin wax embedded sections, although several reports have claimed variable success. ${ }^{1-3}$ Many of the markers currently in routine use such as carcinoembryonic antigen (CEA), ${ }^{4-7}$ Leu M1 (CD15), ${ }^{48}$ and Ber-EP4 ${ }^{9}$ stain carcinomas, particularly adenocarcinoma, but not mesotheliomas, while epithelial membrane antigen (EMA) ${ }^{6}$ and human milk fat globulin 2 (HMFG-2) ${ }^{6}$ stain both, although perhaps in a different pattern. Malignant mesothelioma is, in practice, usually a diagnosis of exclusion rather than positive identification and the use of a panel of immunohistochemical markers is recommended, ${ }^{1011}$ in addition to a mucin stain. Antibodies to CEA are among the most useful in this area, staining carcinomas of tissues that derive embryologically from the foregut. However, ovarian and renal tubular epithelium have a similar embryological derivation to mesothelium and carcinomas of these tissues are often CEA negative.

Recently, two immunohistochemical markers have been claimed to have some mesothelial specificity: HBME-1 (an antibody raised against a suspension of human mesothelioma cells), which reacts with an antigen present in the cell membrane, and thrombomodulin, a transmembrane glycoprotein with a molecular weight of $75 \mathrm{kDa}$, produced in insect cells and found in mesothelial and endothelial cells, among others. This study assessed the value of HBME-1 and antithrombomodulin antibodies in the diagnostic differentiation of pleural malignant mesothelioma from carcinoma. Staining in malignant mesothelioma was compared with staining in cases of reactive pleural mesothelial hyperplasia and carcinoma metastatic to the pleura. Staining of primary ovarian and renal cell carcinomas was also examined, given the problem of marking many of these tumours using traditional techniques and the possibility, due to their embryological relation, that such tumours may express "mesothelial" antigens.

\section{Methods}

Fifty seven cases of malignant mesothelioma were identified from the surgical histopathology files in the department of pathology, Aberdeen Royal Infirmary. Forty seven cases showed purely epithelioid differentiation; the remaining 10 had a sarcomatous appearance, and in three of these this was in addition to epithelioid differentiation-that is, biphasic malignant mesothelioma. Seventeen cases of reactive mesothelial hyperplasia, 23 cases of carcinoma metastatic to the pleura (13 with an origin in the lung and 10 others, including metastases from the breast and gastrointestinal 
Table 1 Immunohistochemical results with $H B M E-1$

\begin{tabular}{lclllllll}
\hline & $-v e$ & $W+$ & $W++$ & $W+++$ & $S+$ & $S++$ & $S+++$ & Total \\
\hline $\begin{array}{l}\text { Malignant } \\
\text { mesothelioma }\end{array}$ & 4 & 3 & 1 & 0 & 4 & 8 & 37 & 57 \\
$\begin{array}{c}\text { Reactive } \\
\text { mesothelial }\end{array}$ & 1 & 1 & 1 & 0 & 1 & 4 & 9 & 17 \\
$\begin{array}{c}\text { hyperplasia } \\
\text { Metastatic } \\
\text { carcinoma in }\end{array}$ & 12 & 0 & 0 & 0 & 3 & 6 & 2 & 23 \\
$\begin{array}{c}\text { pleura } \\
\begin{array}{c}\text { Serous ovarian } \\
\text { carcinoma }\end{array}\end{array}$ & 0 & 0 & 0 & 0 & 0 & 0 & 10 & 10 \\
$\begin{array}{c}\text { Mucinous ovarian } \\
\text { carcinoma }\end{array}$ & 7 & 0 & 0 & 0 & 1 & 1 & 1 & 10 \\
$\begin{array}{c}\text { Renal cell } \\
\text { carcinoma }\end{array}$ & 9 & 1 & 0 & 0 & 0 & 0 & 0 & 10 \\
$\begin{array}{c}\text { Sarcomatoid renal } \\
\text { carcinoma }\end{array}$ & 4 & 0 & 0 & 0 & 0 & 1 & 0 & 5 \\
$\begin{array}{c}\text { Papillary renal } \\
\text { carcinoma }\end{array}$ & 3 & 1 & 0 & 0 & 1 & 0 & 0 & 5 \\
\hline
\end{tabular}

W, weak; S, strong.

Table 2 Immunohistochemical results with thrombomodulin

\begin{tabular}{|c|c|c|c|c|c|c|c|c|}
\hline & $-v e$ & $W+$ & $W++$ & $W+++$ & $S+$ & $S++$ & $S+++$ & Total \\
\hline $\begin{array}{l}\text { Malignant } \\
\text { mesothelioma }\end{array}$ & 8 & 5 & 3 & 0 & 12 & 20 & 9 & 57 \\
\hline $\begin{array}{l}\text { Reactive } \\
\text { mesothelial } \\
\text { hyperplasia }\end{array}$ & 2 & 5 & 6 & 0 & 2 & 2 & 0 & 17 \\
\hline $\begin{array}{l}\text { Metastatic } \\
\text { carcinoma in } \\
\text { pleura }\end{array}$ & 17 & 4 & 0 & 0 & 2 & 0 & 0 & 23 \\
\hline $\begin{array}{l}\text { Serous ovarian } \\
\text { carcinoma }\end{array}$ & 10 & 0 & 0 & 0 & 0 & 0 & 0 & 10 \\
\hline $\begin{array}{l}\text { Mucinous ovarian } \\
\text { carcinoma }\end{array}$ & 9 & 0 & 0 & 0 & 0 & 0 & 1 & 10 \\
\hline $\begin{array}{l}\text { Renal cell } \\
\text { carcinoma }\end{array}$ & 10 & 0 & 0 & 0 & 0 & 0 & 0 & 10 \\
\hline $\begin{array}{l}\text { Sarcomatoid renal } \\
\text { carcinoma }\end{array}$ & 5 & 0 & 0 & 0 & 0 & 0 & 0 & 5 \\
\hline $\begin{array}{l}\text { Papillary renal } \\
\text { carcinoma }\end{array}$ & 4 & 1 & 0 & 0 & 0 & 0 & 0 & 5 \\
\hline
\end{tabular}

W, weak; S, strong. in tables 1 and 2, respectively. No cases demonstrated weak staining in a widespread distribution $(\mathrm{W}+++)$.

HBME-1

Fifty three of 57 malignant mesotheliomas (93\%) stained with HBME-1, 49 (86\%) stained strongly and three quarters of those, $65 \%$ of the total, showed widespread staining. In 10 mesotheliomas with a sarcomatoid element, almost all of the sarcomatoid areas were negative or only weakly and/or focally positive, differing from the positive epithelioid regions in the three biphasic mesotheliomas. In these cases, the positivity of the epithelioid areas was recorded as the result. Sixteen out of 17 cases of reactive mesothelial hyperplasia (94\%) stained with HBME-1, with a wide range of strength and number of cells positive; 14 of $17(82 \%)$ showed strong staining, similar to the pattern seen in mesothelioma.

Twelve of 23 carcinomas metastatic to the pleura were negative with HBME-1, while the 11 positive cases $(48 \%)$ showed a variable amount of strong staining. The metastases from primary lung tumours followed no particular staining pattern, and were as variable in their immunohistochemical staining results as the other metastatic carcinomas. Staining of the primary carcinomas from different sites was variable. All 10 of the serous cystadenocarcinomas of the ovary were strongly positive in a widespread distribution with HBME-1. Seven of the 10 mucinous cystadenocarcinomas of the ovary were negative but three cases demonstrated strong staining with a range of distribution. Nine of 10 renal clear cell carcinomas did not stain with HBME-1, but one case showed weak, focal positivity. Four of five renal carcinomas with a sarcomatoid pattern were negative but one case showed strong, moderate staining. The five renal carcinomas of papillary architecture also demonstrated a mixed result with three being negative and two showing degrees of positive staining. also selected. Each case was reviewed and $5 \mu \mathrm{m}$ sections were cut from representative paraffin embedded blocks and mounted on 3-aminopropyltriethoxysilane coated slides. All tissue had been fixed in $10 \%$ neutral buffered formalin. Microwave heating as the method of antigen unmasking was used. ${ }^{12}$

Immunohistochemistry was carried out using the streptavidin-biotin complex horseradish peroxidase ( $\mathrm{SABC} H \mathrm{HR}$ ) method $^{13}$ with mouse monoclonal antibodies against HBME-1 (Dako M3505; High Wycombe, Bucks, UK), dilution $1 / 80$, and antithrombomodulin (Dako M0617), dilution 1/10, using appropriate positive controls. The negative control for each case used Tris buffered saline.

Immunoreactivity was scored by two observers as negative (no immunostaining) or positive. Positive results were evaluated as strong or weak. The percentage of immunostained cells in the population of interest was recorded as follows: + (focal, $<5 \%$ of cells); ++ (moderate, $5-70 \%$ of cells); +++ (widespread, $>70 \%$ of cells).

\section{Results}

The immunohistochemical results with HBME-1 and antithrombomodulin are shown

\section{ANTITHROMBOMODULIN}

Forty nine of 57 malignant mesotheliomas $(86 \%)$ stained with antithrombomodulin. As with HBME-1, the largest proportion (41 of $57,72 \%$ ) were strongly positive but the density of strongly stained cells was more variable, with the strong, moderate pattern being the most common (fig 1). The sarcomatoid areas of the malignant mesotheliomas showed similar results to those seen with HBME-1; most did not stain. Nearly all cases of reactive mesothelial hyperplasia stained $(15$ of $17,88 \%$ ) with a range of positive cell density. None showed strong, widespread staining, compared to nine out of $17(53 \%)$ cases staining strongly in a widespread distribution with HBME-1.

Overall, the carcinomas showed much less staining with antithrombomodulin than with HBME-1. Seventeen of the 23 metastatic carcinomas did not stain with antithrombomodulin. Those that did (six cases, $26 \%$ ) were only focally positive (strong and weak staining). All 10 ovarian serous cystadenocarcinomas were negative, as were nine of 10 mucinous 


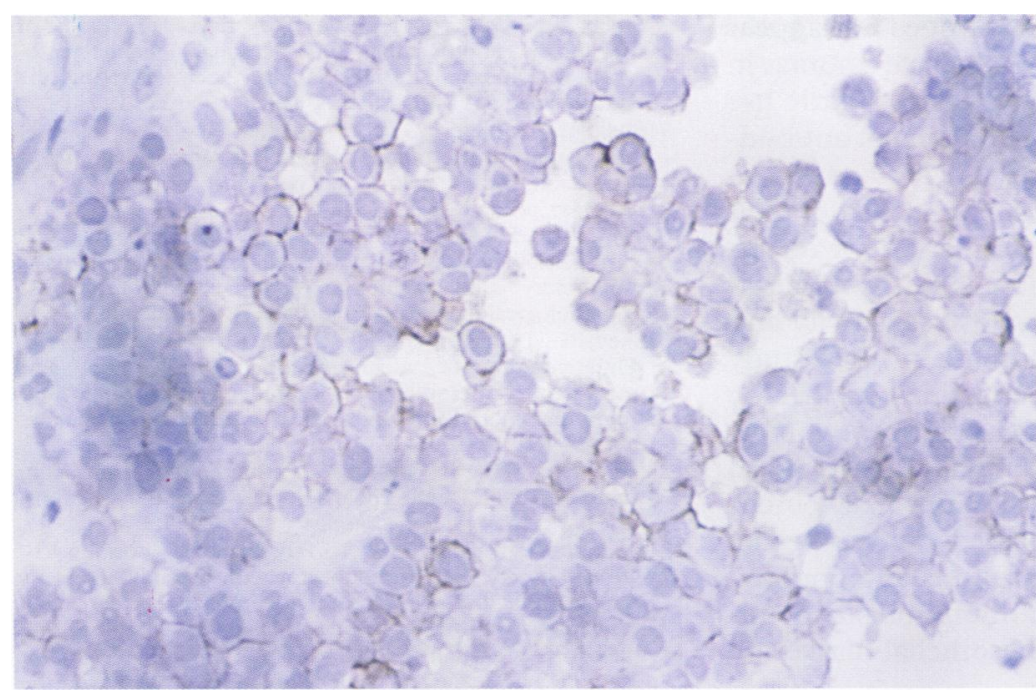

Figure 1 An example of strong, moderate staining by antithrombomodulin. Note that, typically, not all of the cells show staining ( $A A B C$ method).

cystadenocarcinomas, with only one demonstrating a strong, widespread pattern. Nineteen of 20 renal carcinomas were also negative with antithrombomodulin, with only one carcinoma (of papillary architecture) weakly and focally positive.

The pattern of staining with both antibodies was membranous, with occasional additional cytoplasmic staining. There appeared to be no difference in the cellular localisation of staining between mesothelioma, carcinoma, or reactive hyperplasia.

\section{Discussion}

This study has shown that HBME-1 strongly stained $86 \%$ of malignant mesothelioma cases and $82 \%$ of reactive mesothelial cases. However, HBME-1 was not mesothelial specific because $48 \%$ of carcinomas metastatic to the pleura were also stained, each of them strongly. All serous ovarian carcinomas stained strongly in a widespread distribution.

In comparison, antithrombomodulin was slightly less sensitive for malignant mesothelioma ( $72 \%$ of cases stained strongly positive) and had a tendency for moderate or focal rather than widespread distribution. However, it was more specific because $74 \%$ of cases of metastatic carcinoma were negative and none showed strong and moderate or widespread staining. Of the cases of renal and ovarian carcinoma, $95 \%$ of the combined total were negative with antithrombomodulin. Antithrombomodulin also stained $88 \%$ of cases of reactive mesothelial hyperplasia.

In 1992, the original report on HBME-1 by Sheibani et $\mathrm{al}^{14}$ described staining in the majority of cases of epithelial-type malignant mesothelioma, often with a membranous quality, and a few adenocarcinomas also showed staining. This painted an optimistic future for HBME-1, but the lack of specificity for mesothelioma has been documented in our study and in several others, ${ }^{15-17}$ where up to $72 \%$ of pulmonary adenocarcinomas were positive with HBME-1. ${ }^{17}$ Miettinen and Kovatich $^{15}$ also found ovarian serous carcinomas to be positive consistently, but all renal carcinomas that they studied were negative. These authors suggested, with some reservations, that HBME-1 would be useful in an antibody panel. Our results and those of other studies ${ }^{16}{ }^{17}$ would not support this view.

Attanoos and coworkers ${ }^{17}$ found that antithrombomodulin had a $52 \%$ sensitivity for mesothelioma and only two out of 32 adenocarcinomas were positive, while Collins et al ${ }^{18}$ found antithrombomodulin to have a $100 \%$ sensitivity and $92 \%$ specificity for mesothelioma. We have shown an $86 \%$ sensitivity for mesothelioma and agree that thrombomodulin has a high enough specificity to differentiate between epithelial-type malignant mesothelioma and adenocarcinoma. In another study, thrombomodulin staining of mesothelioma and renal cell carcinoma was compared. ${ }^{19}$ Again, sensitivity for mesothelioma was lower than ours (11 of 20 cases were positive) but they showed only one case of renal carcinoma to be positive with thrombomodulin, as we did. This study concurs with our view that antithrombomodulin does not have a role in distinguishing between sarcomatoid renal carcinoma and sarcomatoid malignant mesothelioma, as both entities fail to stain.

Thrombomodulin is mesothelial rather than mesothelioma specific, because most cases of reactive and malignant mesothelium are labelled by antithrombomodulin. We agree with Collins and colleagues, ${ }^{18}$ who suggest that this antibody has no discriminating role in separating benign from malignant mesothelial cells. The tendency for strong staining in malignant mesothelial cells and weaker staining in reactive cells is probably not useful in the diagnostic situation.

In a study of serous effusions, different patterns of staining with antithrombomodulin were found for malignant mesothelioma, reactive mesothelial cases, and carcinomatous fluids; staining was described as thick membranous staining, thin membranous, and cytoplasmic, respectively. ${ }^{20}$ Thirty nine per cent of carcinomatous fluids were positive in this study, a lower specificity than we demonstrated. The differences in specificity and staining patterns may be due to the medium in which the cells are presented and differences in antigen unmasking techniques.

Antithrombomodulin stained vascular endothelium. Consequently, tumours such as renal cell carcinoma, which has a prominent vascular pattern, were rather difficult to score. In addition, antithrombomodulin may show only focal or weak staining, which is difficult to identify on low power scanning. This could lead to false negative scoring on small tissue samples. Furthermore, in malignant mesotheliomas demonstrating strong but focal staining with antithrombomodulin, there was often a moderate but weak underlying pattern of staining in tumour cells not showing strong staining. Haemosiderin and occasional necrotic cells also stained positively with antithrombomodulin, as did renal tubules which had a granular positivity.

We conclude, therefore, that although HBME-1 is sensitive at decorating benign and 
malignant mesothelial cells, it does not appear to be specific, because $44 \%$ of all carcinomas in this study stained positively. There is little use for HBME-1 as a marker in a panel of antibodies designed for the differential diagnosis of malignancy in the pleura. We consider that thrombomodulin is a useful marker for distinguishing malignant mesothelioma from carcinoma, given that it has an $86 \%$ sensitivity and only $13 \%$ of all carcinomas in this study stained; its usefulness is enhanced further by the fact that it does not significantly label tumours of organs with a similar embryological derivation to that of mesothelium that, potentially, might have expressed "mesothelial" markers. Antithrombomodulin is an appropriate antibody for use in an immunohistochemical panel as a "positive" mesothelial marker.

1 Edwards C, Oates J. OV 632 and MOC 31 in the diagnosis of mesothelioma and adenocarcinoma: an assessment of their use in formalin fixed and paraftin

2 Skov BG, Stahel RA, Hirsch F. Mel-antibody labelling of primary bronchogenic tumours and extrapulmonary malignancies. Lung Cancer 1994;11:221-7.

3 Doglioni C, Dei Tos AP, Laurino L, Iuzzolino P, Chiarelli C Celio MR, et al. Calretinin: a novel immunocytochemical marker for mesothelioma. Am f Surg Pathol 1996;20:103746.

4 Ordonez NG. The immunohistochemical diagnosis of mesothelioma. Am $\mathcal{F}$ Surg Pathol 1989;13:276-91.

5 Brown RW, Clark GM, Tandon AK, Allred DC. Multiplemarker immunohistochemical phenotypes distinguishing marker immunohistochemical phenotypes distinguishing malignant pleural mesothelioma from p

6 Cibas ES, Corson JM, Pinkus GS. The distinction of adenocarcinoma from malignant mesothelioma in cel blocks of effusions: the role of routine mucin histochemistry and immunohistochemical assessment of carcinoembryonic antigen, keratin proteins, epithelial membrane antigen, and milk fat globule-derived antigen. Hum Pathol 1987;18:67-74.

7 Holden J, Churg A. Immunohistochemical staining for keratin and carcinoembryonic antigen in the diagnosis of malignant mesothelioma. Am f Surg Pathol 1984;8:277-9.
8 Sheibani K, Battifora H, Burke JS. Antigenic phenotype of malignant mesotheliomas and pulmonary adenocarcinomas. An immunohistologic analysis demonstrating the value of Leu M1 antigen. Am f Pathol 1986;123:212-19.

9 Sheibani K, Shin SS, Kezirian J, Weiss LM. Ber-EP4 antibody as a discriminant in the differential diagnosis of malignant mesothelioma versus adenocarcinoma. Am $\mathfrak{f}$ Surg Pathol 1991;15:779-84.

10 Skov BG, Lauritzen AF, Hirsch F, Nielsen HW. The histopathological diagnosis of malignant mesothelioma v. pulmonary adenocarcinoma: reproducibility of the histopathological diagnosis. Histopathology 1994;24:553-7.

11 Skov BG, Lauritzen AF, Hirsch FR, Skov T, Nielsen HW. Differentiation of adenocarcinoma of the lung and malignant mesothelioma: predictive value and reproducibility of immunoreactive antibodies. Histopathology 1994;25:431-7.

12 King G. Microwave heating as a method of unmasking immunoglobulin light chain antigen in paraffin sections. UK NEQAS Immunocytochemistry News 1994;3:1 1-12.

13 Coutinho HB, King G, Sewell HF, Tighe P, Coutinho VB, Robalinho TI, et al. Immunocytochemical study of Peyer's patches follicular-associated epithelium in the marsupial, Didelphis albiventris. Dev Comp Immunol 1993;17:537-48.

14 Sheibani K, Esteban JM, Bailey A, Battifora H, Weiss LM. Immunopathologic and molecular studies as an aid to the diagnosis of malignant mesothelioma. Hum Pathol 1992;23: $107-16$.

15 Miettinen M, Kovatich AJ. HBME-1 a monoclonal antibody useful in the differential diagnosis of mesothelioma, adenocarcinoma, and soft-tissue and bone tumors. Appl Immunohistochem 1995;3:115-22.

16 Bateman AC, Al-Talib RK, Newman T, Williams JH, Herbert A. Immunohistochemical phenotype of malignant mesothelioma: predictive value of CA 125 and HBME-1 expression. Histopathology 1997;30:49-56.

17 Attanoos RL, Goddard H, Gibbs AR. Mesotheliomabinding antibodies: thrombomodulin, OV 632 and HBME-1 and their use in the diagnosis of malignant mesothelioma. Histopathology 1996;29:209-15.

18 Collins CL, Ordonez NG, Schaefer R, Cook CD, Xie SS, Granger J, et al. Thrombomodulin expression in malignant pleural mesothelioma and pulmonary adenocarcinoma. Am ₹ Pathol 1992;141:827-33.

19 Attanoos RL, Goddard H, Thomas ND, Jasani B, Gibbs AR. A comparative immunohistochemical study of malignant mesothelioma and renal cell carcinoma: the diagnostic utility of Leu-M1, Ber EP4, Tamm Horsfall protein and utility of Leu-M1, Ber EP4, Tamm Horsfall prote
thrombomodulin. Histopathology 1995;27:361-6.

20 Ascoli V, Scalzo CC, Taccogna S, Nardi F. The diagnostic value of thrombomodulin immunolocalization in serous effusions. Arch Pathol Lab Med 1995;119:1136-40. 